\title{
REGENERAÇÃO NATURAL DE UMA ÁREA dE CAATINGA NO SERTÃo PERNAMBUCANO, NORDESTE DO BRASIL
}

\author{
Francisco Tarcisio Alves Junior ${ }^{1}$, Rinaldo Luiz Caraciolo Ferreira², José Antônio Aleixo da Silva ${ }^{3}$, \\ Luiz Carlos Marangon ${ }^{4}$, German Hugo Guttierez Cespedes ${ }^{5}$
}

(recebido: 3 de fevereiro de 2011; aceito: 25 de janeiro de 2013)

\begin{abstract}
RESUMO: Objetivou-se analisar a estrutura da regeneração natural das espécies lenhosas de área de caatinga no município de Floresta-PE com importância madeireira, buscando com informação para o manejo florestal sustentado dessa formação. O inventário florestal foi realizado em uma área de cerca de 50 ha com vegetação de caatinga na fazenda Itapemirim ( $8^{\circ} 30^{\prime} 37^{\prime}$ ' S e $37^{\circ} 59^{\prime} 07^{\prime}$ ' W), município de Floresta-PE. Em 40 unidades amostrais de 5 x $5 \mathrm{~m}$, foram mensurados os indivíduos em quatro classes de tamanho: classe $1=20-50 \mathrm{~cm}$; classe $2=51-100 \mathrm{~cm}$; classe $3=101-150 \mathrm{~cm}$; classe $4=$ maior que $151 \mathrm{~cm}$ e menores que $6 \mathrm{~cm}$ de CAP. Em 37 parcelas, havia indivíduos dentro do nível de inclusão e foram identificadas 15 espécies pertencentes a sete famílias botânicas, destacando-se Euphorbiaceae, Anacardiaceae e Fabaceae. O Índice de diversidade de Shannon-Weaver (H') foi de 1,91 nats.ind. ${ }^{-1}$, o Índice de uniformidade de Pielou (E) igual a de 0,71. A área apresentou densidade estimada de 2.080 ind. ha ${ }^{-1}$. A distribuição espacial evidenciou as características da maioria das espécies, de ocorrerem agrupadas ou com tendência ao agrupamento. As espécies Bauhinia cheilanta e Poincianella bracteosa apresentaram maiores valores de densidade, além de estarem presentes em todas as classes de regeneração natural, constituindo indícios de que essas espécies apresentam potencial de se estabelecerem no local e serem as possíveis espécies dominantes quando chegarem à fase adulta.
\end{abstract}

Palavras-chave: Diversidade, distribuição especial, manejo florestal.

\section{NATURAL REGENERATION OF AN AREA OF CAATINGA VEGETATION IN PERNAMBUCO STATE, NORTHEASTERN BRAZIL}

\begin{abstract}
This study aimed to analyze the structure of natural regeneration of economically important woody species of Caatinga in the municipality of Floresta-PE, assisting with information for sustainable forest management of the forest physiognomy. The forest inventory was conducted in an area of about 50 ha of savanna vegetation ( $8^{\circ} 30^{\prime} 37^{\prime \prime} \mathrm{S}$ and $37^{\circ} 59^{\prime} 07^{\prime}$ " W), municipality of Floresta$P E$. With 40 sampling units of $5 \times 5 \mathrm{~m}$, were measured the subjects into four classes: class $1=20-50 \mathrm{~cm}$, class $2=51-100 \mathrm{~cm}$, class $3=101-150 \mathrm{~cm}$, class $4=$ greater than $151 \mathrm{~cm}$ and less than $6 \mathrm{~cm}$ in circumference of the base height. In only 37 plots had individuals in the level of inclusion and were identified 15 species belonging to seven botanical families, especially Euphorbiaceae, Fabaceae and Anacardiaceae. The diversity of Shannon-Weaver index (H') was 1.91 nats.ind.-1, the uniformity of Pielou index (E) equals 0.71 . The area presented estimated density of 2,080 ind. ha ${ }^{-1}$. The spatial distribution showed that the characteristics of most species occur in group or with a tendency to grouping. The species Bauhinia cheilanta and Poincianella bracteosa showed higher density values and were present in all classes of natural regeneration, providing evidence that they have potential to establish themselves on the area and possibly will be the dominant species after reaching adulthood.
\end{abstract}

Key words: Diversity, distribution special, forest management.

\section{INTRODUÇÃO}

Cobrindo $55 \%$ dos $1.548 .672 \mathrm{~km}^{2}$ da área da região Nordeste do Brasil, na porção semiárida (RODAL et al., 2002), a caatinga é a vegetação predominante (INSTITUTO BRASILEIRO DE GEOGRAFIA E
ESTATÍSTICA - IBGE, 2005), está compreendida entre os paralelos de $2^{\circ} 54^{\prime} \mathrm{S}$ a $17^{\circ} 21^{\prime} \mathrm{S}$ e envolve áreas dos Estados do Ceará, do Rio Grande do Norte, da Paraíba, de Pernambuco, de Alagoas, de Sergipe, do Piauí, da Bahia e de Minas Gerais (ANDRADE et al., 2005). Atualmente, ainda quase $40 \%$ da área original são recobertas de

\footnotetext{
${ }^{1}$ Engenheiro de Produção, Doutor em Ciências Florestais - Universidade Federal Rural de Pernambuco/UFRPE - Rua Manoel de Medeiros, s/n, Dois Irmãos - 52.171-900 - Recife, PE, Brasil-tarcisioalvesjr@yahoo.com.br

${ }^{2}$ Engenheiro Florestal, Professor Doutor em Ciência Florestal - Universidade Federal Rural de Pernambuco/UFRPE - Departamento de Ciência Florestal - Rua Manoel de Medeiros, s/n, Dois Irmãos - 52.171-900 - Recife, PE, Brasil - rinaldo@dcfl.ufrpe.br

${ }^{3}$ Engenheiro Agrônomo, Professor Doutor em Biometria e Manejo Florestal - Universidade Federal Rural de Pernambuco/UFRPE - Departamento de Ciência Florestal - Rua Manoel de Medeiros, s/n, Dois Irmãos - 52.171-900 - Recife, PE, Brasil - jaaleixo@uol.com.br

${ }^{4}$ Engenheiro Florestal, Professor Doutor em Ecologia e Recursos Naturais - Universidade Federal Rural de Pernambuco/UFRPE - Departamento de Ciência Florestal - Rua Manoel de Medeiros, s/n, Dois Irmãos - 52.171-900 - Recife, PE, Brasil - marangon@dcfl.ufrpe.br

${ }^{5}$ Engenheiro Florestal, Pesquisador Mestre em Genética - Empresa Agroindustrial Excelsior S.A./Agrimex - Engenho Itapicirica - 55.900-000 Goiana, PE, Brasil - germanguitierrez@joaosantos.com.br
}

Cerne, Lavras, v. 19, n. 2, p. 229-235, abr./jun. 2013 
vegetação nativa (SILVA; SAMPAIO, 2008). As ameaças à conservação da caatinga devem-se à prática de atividades como contínuos desmatamentos para estabelecer pastagens e utilização de técnicas de irrigação inadequadas; essas práticas intensificam a desertificação; o assoreamento dos rios e aceleram ainda mais o desgaste do solo (LEAL et al., 2005). Esse tipo de exploração em um ambiente pouco conhecido e complexo poderá levar o mesmo a um processo irreversível de degradação (SANTANA; SOUTO, 2006).

No Nordeste, o setor florestal gera cerca de $170 \mathrm{mil}$ empregos diretos e 500 mil indiretos, além de contribuir com $15 \%$ da renda global dos produtores, destaca-se também pela produção de lenha, uma vez que $35 \%$ do seu parque industrial têm a lenha como fonte de energia primária, além de atender à $70 \%$ da demanda energética dos domicílios da região (CAMPELLO et al., 2000).

Sá, Riché e Fotius (2003) indicaram a área denominada de carvoejamento, abrangendo os municípios de Arcoverde, Buique, Tupanatinga, Calumbi, Flores, Betânia, Serra Talhada, Ibimirim, Floresta, Sertânia e Custódia, onde existe inadequada exploração da vegetação da nativa, e deve ser considerada como das áreas prioritária para conservação da caatinga.

Vários estudos de inventários fitossociológicos e florísticos foram realizados nos últimos anos em áreas de caatinga, tendo fornecido importantes informações sobre o número de indivíduos por hectare, a área basal ocupada e riqueza florística. No entanto, existe uma escassez de estudos sobre regeneração natural na vegetação caatinga em Pernambuco. Além disso, tais conhecimentos são essenciais para a elaboração e aplicação correta dos planos de manejo e tratamentos silviculturais permitindo uma exploração racional dessa vegetação.

Diante do exposto, objetivou-se analisar a estrutura da regeneração natural das espécies lenhosas em área de caatinga no município de Floresta-PE com importância madeireira, fornecendo informação para o manejo florestal sustentado dessa formação.

\section{MATERIAL E MÉTODOS}

O inventário florestal foi realizado em uma área de 50 ha com vegetação de caatinga na fazenda Itapemirim ( $8^{\circ} 30^{\prime} 37^{\prime \prime}$ S e $\left.37^{\circ} 59^{\prime} 07^{\prime \prime} \mathrm{W}\right)$, localizada a $70 \mathrm{~km}$ da sede do município de Floresta-PE.

O clima, segundo a classificação de Köppen, é do tipo BSh, semiárido quente, apresentando precipitação média anual de aproximadamente $503 \mathrm{~mm}$, com período chuvoso de dezembro a abril e temperatura média anual de $26,1^{\circ} \mathrm{C}$ (Figura 1 ).

Cerne, Lavras, v. 19, n. 2, p. 229-235, abr./jun. 2013

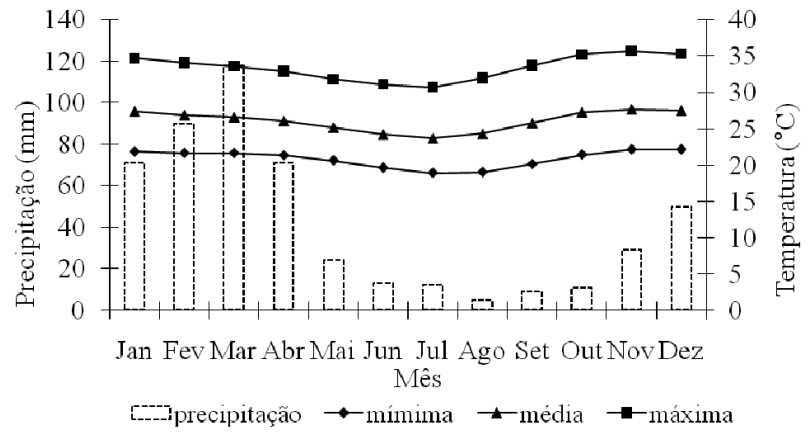

Figura 1 - Médias dos últimos 30 anos da temperatura e precipitação, de janeiro a dezembro, do município de Floresta - PE.

Figure 1 - Averages for the last 30 years of temperature and precipitation from January to December of the municipality of Floresta, Pernambuco state, Brazil.

Fonte: elaborado a partir de dados do Instituto de Tecnologia de Pernambuco - ITEP (2010)

A vegetação da área é do tipo savana-estépica (VELOSO; RANGEL-FILHO; LIMA, 1991), caracterizada por caatinga arbustivo-arbórea, com presença de cactáceas e estrato herbáceo, podendo conter, em alguns locais, macambira (Bromelia laciniosa Mart. ex Schultes f.) e caroá (Neoglaziovia variegata (Arr. Cam.) Mez.).

Foram amostradas 40 parcelas geoferenciadas com dimensões de 5 x $5 \mathrm{~m}$ cada, para os levantamentos dos indivíduos. As parcelas foram demarcadas a partir de $50 \mathrm{~m}$ da borda da vegetação e distanciadas por $80 \mathrm{~m}$ entre si. Em cada parcela, foram mensurados os indivíduos em estágio de regeneração classificados em quatro classes de tamanho: classe $1=20-50 \mathrm{~cm}$; classe $2=51-100 \mathrm{~cm}$; classe $3=101$ $-150 \mathrm{~cm}$; classe $4=$ maior que $151 \mathrm{~cm}$ de altura e menores que $6 \mathrm{~cm}$ de CAP (COMITÊ TÉCNICO CIENTÍFICO DA REDEDE MANEJO FLORESTALDACAATINGA, 2005).

Os indivíduos foram identificados no local pelo o nome vulgar, coletando-se material botânico para posterior identificação, no herbário Sérgio Tavares do Departamento de Ciência Florestal da UFRPE. A sinonímia e a grafia foram realizadas mediante consulta a Forzza et al. (2010), sendo o sistema de classificação adotado o Angiosperm Phylogeny Group - APG (2003).

A Regeneração Natural Total (RNT) foi calculada somando-se as regenerações de cada uma das quatro classes, e dividindo pela quantidade de classe, para obter-se a porcentagem (MUELLER-DOMBOIS; ELLEMBERG, 1974). Para estimar a diversidade florística, foram 
utilizados os índices diversidade de Shannon-Wiener $\left(\mathrm{H}^{\prime}\right)$, Equabilidade de Pielou (J), a Dominância de Simpson (C) e o Coeficiente de Mistura de Jentsch (QM), descritos em Brower e Zarr (1984) e Magurran (1988).

Para verificar o padrão de distribuição espacial das espécies na área, utilizou-se o Índice de Agregação de McGuinnes - IGA (MCGUINNES, 1934). Os dados foram processados com o auxilio do software Mata Nativa (MATA..., 2006).

\section{RESULTADOS E DISCUSSÃO}

Das 40 unidades amostrais, em 37 haviam indivíduos dentro do nível de inclusão no levantamento dos regenerantes. Foram identificadas 15 espécies pertencentes a sete famílias botânicas (Tabela 1). Com esses resultados calculou-se o Índice de diversidade de Shannon-Weaver (H'), que foi de 1,91 nats.ind.-1, o Índice de uniformidade de Pielou $(E)$, com valor de 0,705. Para Índice de dominância de Simpson (D) o valor foi 0,771 e o Coeficiente de Mistura de Jentsch (QM) igual a 0,072.

Comparado ao levantamento das espécies adultas, na mesma área realizado por Alves Júnior (2010), as espécies Varronia leucocephala (Moric.) J.S.Mill., Cynophalla flexuosa (L.) J.Presl, Neocalyptrocalyx longifolium (Mart.) Cornejo \& Iltis, Erytroxylum sp., Cnidosculus bahianus (Ule) Pax \& K. Hoffm., Croton blanchetianus Baill., Manihot glaziovii Muell. Arg., Sapium lanceolatum Huber, Piptadenia stipulacea (Benth.) Ducke, Erythrostemon calycina (Benth.) L.P.Queiroz e Ziziphus joazeiro Mart. não apresentaram indivíduos regenerantes. A maioria dessas espécies demonstraram baixa densidade de indivíduos por hectare, o que pode ter dificultado sua inclusão na amostragem da regeneração. A regeneração natural de espécies arbóreas em uma comunidade florestal depende da produção de sementes, do estabelecimento das plântulas, da sobrevivência das mudas e do recrutamento (YADAVAND; GUPTA, 2009). Para Padilla e Pugnare (2012), a regeneração natural nos ambientes semiáridos geralmente é lenta, pois depende principalmente da precipitação, da dispersão das sementes, da existência de um banco de sementes viáveis no solo e da rebrota de tocos e raízes.

As famílias Euphorbiaceae, Anacardiaceae e Fabaceae corresponderam com a maioria das espécies da área. Essas famílias também se apresentaram como as de maior número de espécies em outros estudos sobre regeneração natural (ANDRADE et al., 2007; PEREIRA et al., 2001).

Tabela 1 - Florística da regeneração natural, em uma área de caatinga, Floresta-PE.

Table 1 - Floristic of natural regeneration of an area of caatinga, Floresta, Pernambuco state, Brazil.

\begin{tabular}{lll}
\hline Família & Espécie & Nome local \\
\hline Anacardiaceae & Myracrodruon urundeuva Allemão & aroeira \\
& Schinopsis brasiliensis Engl. & baraúna \\
& Spondias tuberosa Arruda & umbuzeiro \\
\hline Apocynaceae & Aspidosperma pyrifolium Mart. & pereiro \\
\hline Burseraceae & Commiphora leptophloeos (Mart.) J.B. Gillett & imburana de cambão \\
\hline Combretaceae & Thiloa glaucocarpa (Mart.) Eichl. & sipaúba \\
\hline \multirow{3}{*}{ Euphorbiaceae } & Cnidoscolus phyllacanthus (Müll. Arg.) Pax & faveleira \\
& \& L. Hoffm. & quebra faca \\
& Croton rhamnifolius Willd. & pinhão bravo \\
\hline & Jatropha mollissima (Pohl) Baill. & angico \\
Fabaceae & Anadenanthera colubrina var. cebil (Griseb.) & mororó \\
& Altschul & jurema de embira \\
& Bauhinia cheilanta (Bong). Steud. & jurema preta \\
& Mimosa ophthalmocentra Mart. ex Benth. & catingueira \\
\hline Olacaceae & Mimosa tenuiflora (Willd.) Poir. & ameixa \\
\hline
\end{tabular}

Cerne, Lavras, v. 19, n. 2, p. 229-235, abr./jun. 2013 
Fabricante e Andrade (2008), trabalhando com indivíduos regenerantes na caatinga, no Seridó paraibano encontraram 15 espécies e sete famílias botânicas. Alves et al. (2010), pesquisando regeneração natural em uma área de caatinga em Pombal-PB sob pastejo de bovinos no período seco, identificaram 13 espécies e sete famílias botânicas. Em uma área com três níveis de perturbação, no agreste paraibano, foram encontradas 17 famílias e 26 espécies regenerantes (PEREIRA et al., 2001). Andrade et al. (2007), estudando campos abandonados de sisal (Agave sisalana Perrine ex Engelm.) na Paraíba, a aproximadamente 30 anos, levantou 16 famílias botânicas e 31 espécies.

As espécies que apresentaram maior destaque por classe foram: Bauhinia cheilanta, Poincianella bracteosa e Anadenanthera colubrina var. cebil com $70 \%$ da Regeneração Natural da Classe 1 (RNC1) e Densidade Relativa (DR) de $78,72 \%$ da classe I; Bauhinia cheilanta, Poincianella bracteosa e Jatropha mollissima com $73,50 \%$ da RNC2 e DR de $78,72 \%$ da classe II; Bauhinia cheilanta e Poincianella bracteosa com $77,78 \%$ da RNC3 e DR de $80,56 \%$ da classe III; e
Bauhinia cheilanta, Poincianella bracteosa e Croton rhamnifolius com $72,12 \%$ da RNC4 e DR de $75,00 \%$ da classe IV (Tabela 2).

A espécie Mimosa tenuiflora apresentou indivíduos apenas na primeira classe de tamanho, para Spondias tuberosa e Commiphora leptophloeos foi amostrado um único indivíduo na classe II e III, respectivamente, e Myracrodrun urundeuva e Schinopsis brasiliensis apenas apresentaram indivíduos na classe de maior tamanho. Esse comportamento, demonstra indícios de que algumas espécies da caatinga não dispõem de recrutamentos contínuos.

A distribuição espacial da maioria das espécies, indica tendência ao agrupamento (Tabela 3). Martins et al. (2003) comentaram ser comum a ocorrência dessa distribuição em florestas tropicais, onde espécies mais abundantes surgem agrupadas ou com tendência ao agrupamento. No entanto, deve-se analisar com cautela a classificação das espécies que apresentam poucos indivíduos no inventário.

A área apresentou densidade estimada de 2.080 ind. ha $^{-1}$. Alves et al. (2010) estimaram densidade absoluta de 4.272 ind. ha ${ }^{-1}$, em uma área dominada por Croton blanchetianus.

Tabela 2 - Classes de regeneração natural em uma área de caatinga, Floresta-PE. Em que: N - número de indivíduos; RNC(i) regeneração natural na classe i; RNT - regeneração natural total, em porcentagem.

Table 2 - Classes of natural regeneration of an area of caatinga, Floresta, Pernambuco state, Brazil. Where: N - number of individuals; $R N C(i)$ - natural regeneration in class $i$; $R N T$ - total natural regeneration, in percentage.

\begin{tabular}{lrrrrrr}
\hline Espécie & $\mathrm{N}$ & $\mathrm{RNC1}$ & $\mathrm{RNC} 2$ & $\mathrm{RNC} 3$ & $\mathrm{RNC4}$ & $\mathrm{RNT}(\%)$ \\
\hline Bauhinia cheilanta & 82 & 32,62 & 39,30 & 42,36 & 31,92 & 36,55 \\
Poincianella bracteosa & 49 & 22,81 & 25,17 & 35,42 & 22,79 & 26,55 \\
Anadenanthera colubrina & 17 & 14,48 & 8,19 & 0,00 & 3,17 & 6,46 \\
Jatropha mollissima & 7 & 7,68 & 9,03 & 3,47 & 1,59 & 5,44 \\
Croton rhamnifolius & 15 & 0,00 & 0,00 & 3,47 & 17,40 & 5,22 \\
Aspidosperma pyrifolium & 8 & 5,97 & 7,11 & 3,47 & 1,59 & 4,53 \\
Thiloa glaucocarpa & 8 & 3,84 & 5,18 & 4,86 & 3,17 & 4,26 \\
Mimosa ophthalmocentra & 5 & 3,84 & 0,00 & 0,00 & 5,38 & 2,31 \\
Ximenia americana & 3 & 0,00 & 3,01 & 3,47 & 1,59 & 2,02 \\
Cnidoscolus phyllacanthus & 3 & 3,84 & 0,00 & 0,00 & 2,21 & 1,51 \\
Myracrodrun urundeuva & 5 & 0,00 & 0,00 & 0,00 & 6,01 & 1,50 \\
Mimosa tenuiflora & 2 & 4,91 & 0,00 & 0,00 & 0,00 & 1,23 \\
Commiphora leptophloeos & 1 & 0,00 & 0,00 & 3,47 & 0,00 & 0,87 \\
Schinopsis brasiliensis & 2 & 0,00 & 0,00 & 0,00 & 3,17 & 0,79 \\
Spondias tuberosa & 1 & 0,00 & 3,01 & 0,00 & 0,00 & 0,75 \\
\hline Total & 208 & 100,00 & 100,00 & 100,00 & 100,00 & 100,00 \\
\hline
\end{tabular}

Cerne, Lavras, v. 19, n. 2, p. 229-235, abr./jun. 2013 
Tabela 3 - Distribuição espacial das espécies amostradas na regeneração natural de uma área de caatinga, Floresta-PE. Em que: N: número de indivíduos; IGA: Índice de Agregação de McGuinnes.

Table 3 - Spatial distribution of the species in natural regeneration of an area of caatinga, Floresta, Pernambuco state, Brazil. Where: N: number of individuals; IGA: Aggregation McGuinness Index.

\begin{tabular}{lrc}
\hline Espécies & N & Padrão - IGA \\
\hline Anadenanthera colubrina & 17 & agregado \\
Aspidosperma pyrifolium & 8 & tende ao agrupamento \\
Bauhinia cheilanta & 82 & agregado \\
Cnidoscolus phyllacanthus & 3 & tende ao agrupamento \\
Commiphora leptophloeos & 1 & uniforme \\
Croton rhamnifolius & 15 & tende ao agrupamento \\
Jatropha mollissima & 7 & tende ao agrupamento \\
Mimosa ophthalmocentra & 5 & tende ao agrupamento \\
Mimosa tenuiflora & 2 & tende ao agrupamento \\
Myracrodrun urundeuva & 5 & tende ao agrupamento \\
Poincianella bracteosa & 50 & tende ao agrupamento \\
Schinopsis brasiliensis & 2 & uniforme \\
Spondias tuberosa & 1 & uniforme \\
Thiloa glaucocarpa & 8 & agregado \\
Ximenia americana & 3 & agregado \\
\hline
\end{tabular}

Fabricante e Andrade (2008), no município de Santa Luzia no Seridó, estimaram 2.823 ind.ha-1 ${ }^{-1}$ Pereira et al. (2001), pesquisando no município de Areias, no agreste do estado da Paraíba, identificaram 6.750, 5.500 e 5.100 ind.ha $^{-1}$ para os ambientes I, II e III, respectivamente, com níveis de perturbação crescentes.

Analisando as densidades das espécies distribuídas nas classes de altura, os indivíduos apresentaram uma distribuição semelhante nas duas classe de altura inicial e um aumento na classe IV (Figura 2). A menor quantidade no número de indivíduos das menores classes pode ser explicada pelo fato de ser mais suscetível a mortalidade e ao ingresso, quando a variação no número de indivíduos das classes maiores ocorre pelo o fato de os indivíduos mudarem de classe.

Sampaio et al. (1998), pesquisando uma área de caatinga em Serra talhada, após corte e queima, verificou que a espécie Croton blanchetianus sobressaiu entre as demais espécies no local. O mesmo ocorrendo em uma área de caatinga antropizada, exploração de madeira e retirada

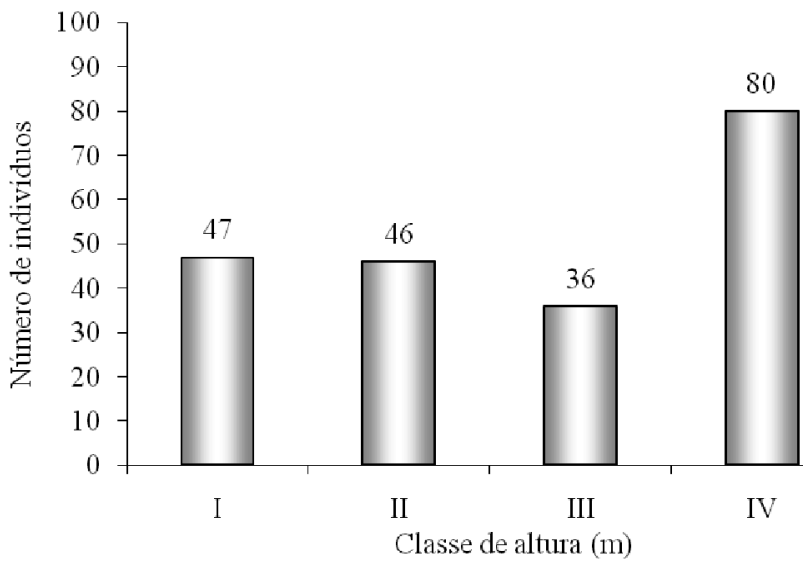

Figura 2 - Número de indivíduos amostrados por classe de altura da regeneração natural em uma área de caatinga, Floresta-PE. Em que: classe I $=20-50 \mathrm{~cm}$; classe II $=51-100 \mathrm{~cm}$; classe $\mathrm{III}=101-150 \mathrm{~cm}$; classe IV = maior que $151 \mathrm{~cm}$ e menores que $6 \mathrm{~cm}$ de circunferência a altura do peito (CAP).

Figure 2-Number of individuals sampled by size class of natural regeneration in an area of caatinga, Floresta, Pernambuco state, Brazil. Where: class $1=20-50 \mathrm{~cm}$, class $2=51-100 \mathrm{~cm}$, class $3=101-150 \mathrm{~cm}$, class $4=$ greater than $151 \mathrm{~cm}$ and less than 6 $\mathrm{cm}$ in circumference of the breast height.

do estrato inferior formado por Bromelia laciniosa Mart. ex Shult. (macambira) foi totalmente eliminado para ser fornecido ao gado (PEREIRA et al., 2001).

\section{CONCLUSÕES}

Algumas espécies do estrato adulto não apresentaram indivíduos regenerantes e necessitam de um melhor acompanhamento silvicultural, quando submetidas à exploração florestal.

Considerando todas as classes de regeneração, as espécies Bauhinia cheilanta e Poincianella bracteosa, apresentaram maiores valores de densidade, além de estarem presentes em todas as classes de regenerarão natural, constituindo indícios de que essas espécies apresentam potencial de se estabelecerem no local e serem as possíveis espécies dominantes quando chegarem à fase adulta.

\section{REFERÊNCIAS}

ALVES, L. S. et al. Regeneração natural em uma área de caatinga situada no município de Pombal-PB, Brasil. Revista Verde de Agroecologia e Desenvolvimento Sustentável, Mossoró, v. 5, n. 2, p. 152-168, 2010.

Cerne, Lavras, v. 19, n. 2, p. 229-235, abr./jun. 2013 
ALVES JÚNIOR, F. T. Estrutura, biomassa e volumetria de uma área de caatinga, Floresta, PE. 2010. 123 f. Tese (Doutorado em Ciências Florestais) - Universidade Federal Rural de Pernambuco, Recife, 2010.

ANDRADE, L. A. et al. Análise da cobertura de duas fitofisionomias de caatinga, com diferentes históricos de uso, no município de São João do Cariri, estado da Paraíba. Cerne, Lavras, v. 11, n. 3, p. 253-260, 2005.

ANDRADE, L. A. et al. Análise da vegetação sucessional em campos abandonados no agreste paraibano. Revista Brasileira de Ciências Agrárias, Recife, v. 2, n. 2, p. 135-142, 2007.

ANGIOSPERM PHYLOGENY GROUP. An update of the Angiosperm Phylogeny Group classification for the orders and families of flowering plants: APG II. Botanical Journal of the Linnean Society, London, v. 141, n. 4, p. 399-436, 2003.

BROWER, J. E.; ZARR, J. H. Field and laboratory methods for general ecology. $2^{\text {nd }}$ ed. Iowa: W. C. Brown, 1984. 226 p.

CAMPELLO, F. C. B. et al. Avaliação dos recursos florestais da área de proteção ambiental Chapada do Araripe. Crato: MMA, 2000. 68 p.

COMITÊ TÉCNICO CIENTÍFICO DA REDE DE MANEJO FLORESTAL DA CAATINGA. Rede de manejo florestal da Caatinga: protocolo de medições de parcelas permanentes/ Comitê Técnico Científico. Recife: Associação Plantas do Nordeste, 2005. 21 p.

FABRICANTE, J. R.; ANDRADE, L. A. Análise estrutural de um remanescente de caatinga no Seridó paraibano. Oecologia Brasiliensis, Rio de Janeiro, v. 11, n. 3, p. 341-349, 2008.

FORZZA, R. C. et al. Lista de espécies da flora do Brasil. Rio de Janeiro: Jardim Botânico do Rio de Janeiro, 2010. Disponível em: <http://floradobrasil.jbrj.gov.br/2010/>. Acesso em: 11 out. 2010.

INSTITUTO BRASILEIRO DE GEOGRAFIA E ESTATÍSTICA. Mapas de biomas e vegetação. Rio de Janeiro, 2005. Disponível em: <http://www.ibge.gov.br $>$. Acesso em: 2 nov. 2008.

INSTITUTO DE TECNOLOGIA DE PERNAMBUCO. Climatologia. Disponível em: $<$ http://www.itep.br/LAMEPE. asp>. Acesso em: 25 out. 2010.

Cerne, Lavras, v. 19, n. 2, p. 229-235, abr./jun. 2013
LEAL, I. R. et al. Mudando o curso da conservação da biodiversidade na Caatinga do Nordeste do Brasil. Megadiversidade, Belo Horizonte, v. 1, n. 1, p. 139-146, 2005.

MAGURRAN, A. E. Ecological diversity and its measurement. Princeton: Princeton University, 1988. 192 p.

MARTINS, S. S. et al. Efeito da exploração florestal seletiva em uma floresta estacional semidecidual. Revista Árvore, Viçosa, v. 27, n. 1, p. 65-70, jan./fev. 2003.

MATA nativa 2: manual do usuário. Viçosa, MG: CIENTEC, 2006. 295 p.

MCGUINNES, W. G. The relationship between frequency index and abundance as applied to plant populations in a semi-arid region. Ecology, Durham, v. 16, p. 263-282, 1934.

MUELlER-DOMBOIS, D.; ELlEMBERG, H. Aims and methods of vegetation ecology. New York: Wiley, 1974. $547 \mathrm{p}$.

PADILLA, F. M.; PUGNAIRE, F. I. Sucesión y restauración em ambientes semiáridos. Disponível em: $<$ http//www.aeet. org/ecossistemas>. Acesso em: 14 jun. 2012.

PEREIRA, I. M. et al. Regeneração natural em um remanescente de caatinga sob diferentes níveis de perturbação, no agreste paraibano. Acta Botânica Brasílica, São Paulo, v. 15, n. 3, p. 431-426, 2001.

RODAL, M. J. N. et al. A vegetação do bioma Caatinga. In: SAMPAIO, E. V. S. B. et al. (Org.). Vegetação e flora da caatinga. Recife: Associação Plantas do Nordeste, 2002. p. 11-24.

SÁ, I. B.; RICHÉ, G. R.; FOTIUS, G. A. Fatores abióticos: áreas e ações prioritárias para conservação da caatinga. In: SILVA, J. M. C. et al. (Org.). Biodiversidade da caatinga: e ações prioritárias para conservação. Brasília: MMA, 2003. p. 37-44.

SAMPAIO, E. V. S. B. et al. Regeneração da vegetação de caatinga após corte e queima, em Serra Talhada, PE. Pesquisa Agropecuária Brasileira, Brasília, v. 33, n. 5, p. 621-632, maio 1998. 
SANTANA, J. A. S.; SOUTO, J. S. Diversidade e estrutura fitossociológica da caatinga na estação ecológica do Seridó, RN. Revista de Biologia e Ciências da Terra, Campina Grande, v. 6 , n. 2, p. 232-242, 2006.

SILVA, G. C.; SAMPAIO, E. V. S. B. Biomassas de partes aéreas em plantas da caatinga. Revista Árvore, Viçosa, v. 32, n. 3, p. 567-575, maio/jun. 2008.
VELOSO, H. P.; RANGEL-FILHO, A. L. R.; LIMA, J. C. A. Classificação da vegetação brasileira, adaptada a um sistema universal. Rio de Janeiro: IBGE, 1991. 123 p.

YADAVAND, A. S.; GUPTA, S. K. Natural regeneration of tree species in a tropical dry deciduous thorn forest in Rajasthan, India. National Institute of Ecology, New Delhi, v. 20, p. 5-14, 2009.

Cerne, Lavras, v. 19, n. 2, p. 229-235, abr./jun. 2013 
